\title{
Study of Sigma-Delta Modulator for Underwater Acoustic Amplifier
}

\author{
Yongheng Wang ${ }^{1, a}$, Dajun Sun ${ }^{1, b}$ \\ 1. College of Underwater Acoustic Engineering, Harbin Engineering University \\ Harbin, China \\ awangyongheng@hrbeu.edu.cn, bsundajun@hrbeu.edu.cn
}

Keywords: sonar; underwater acoustic amplifier; Sigma-Delta modulator; noise shaping; match filtering

\begin{abstract}
This paper is briefly discussed the theory of Sigma-Delta modulator, analyzed the performance of L-order single bit Sigma-Delta modulator. A system modeling for $2^{\text {nd }}$ order SigmaDelta modulator is simulated. Under laboratory conditions, An acoustic amplifier with Sigma-Delta modulation and PWM modulation is constructed, combined the matching theory, the impedance matching for acoustic transducer is implemented. The test results show that: Sigma-Delta modulation technique can be used for acoustic amplifier. Compared with PWM modulator, SigmaDelta modulator has the features of high output signal-to-noise ratio (SNR), high work efficiency and low total harmonic distortion (THD) and has splendid application value.
\end{abstract}

\section{Introduction}

With the development of ocean technology, sonar as the ocean development indispensable equipment attracted more and more attention. At present, the underwater robot, UUV, buoy system and other sonar platform, especially the closed system using battery, have more limitation about the size, weight and power consumption. In order to adapt the actual demand of sonar devices, miniaturized becomes the trend of the development of sonar system [1].

Acoustic amplifier is an important part of the sonar system. Its performance directly affects the working distance, weight, size and power loss of the sonar system. The category of the acoustic amplifier mainly includes linear power amplifier and nonlinear power amplifier. For the conventional sonar systems, using the traditional class $\mathrm{AB}$ power amplifier to transmit acoustic signal in order to ensure the signal linearity, the amplifier has a maximum theoretical efficiency of $78.5 \%$, however, the actual work efficiency is between $50 \%$ and $70 \%$, which cause a series of sonar system problems in power consumption, heat dissipation, size and weight. Class D power amplifier working in switching mode, the theory of power conversion efficiency is $100 \%$, the typical value is $85 \%$, which can effectively reduce the size and weight of power amplifier and reduce the sonar system power requirements. Therefore, the class $\mathrm{D}$ power amplifier is widely used in sonar system [2].

At present, the class D power amplifier is widely used in audio equipment. The modulation technique is used for audio signal processing. The Pulse-Width Modulation (PWM) and PulseDensity Modulation (PDM) are commonly used in class D power amplifier. In actual application, the PWM modulator has the advantages of simple structure, high output power and high stability, and also has the disadvantage of high harmonic distortion and high EMI [3] [4].

In underwater acoustic communication equipment, the transmitted signal required the amplifier has a low distortion, so the class D power amplifier is restricted, and class AB power amplifier is retained. However, the Sigma-Delta modulation technology which has noise shaping performance can reduce the harmonic distortion of the class $\mathrm{D}$ amplifier.

\section{Sigma-Delta modulation technology}

Sigma-Delta modulation technology has developed with several decades. the theory tends to be mature. At present, the Sigma-Delta modulation technology is widely used in high precision ADC and DAC. Because of the performance of high precision and easy implementation of CMOS VLSI technology, the Sigma-Delta ADC/DAC is attracted the researchers, and became a direction of the 
embedded ADC/DAC integrated circuit design.

Now, TI Company, Maxim Company, National Semiconductor and other internationally wellknown semiconductor manufacturers have released products which including high precision analogto-digital converter, high precision digital to analog converter and Sigma-Delta class D power amplifier. The next part will give the basic structure of an L-order Sigma-Delta modulator and analysis the basic performance.

A. L-order Single Bit Sigma-Delta Modulator.

The modulator consists of L integrators and one quantizer, the output of quantizer feedback to the each integrator, completed the loop filter. $\mathrm{X}[\mathrm{n}]$ is the input signal, $\mathrm{E}[\mathrm{n}]$ is quantization noise, $\mathrm{Y}[\mathrm{n}]$ is modulated output signal.

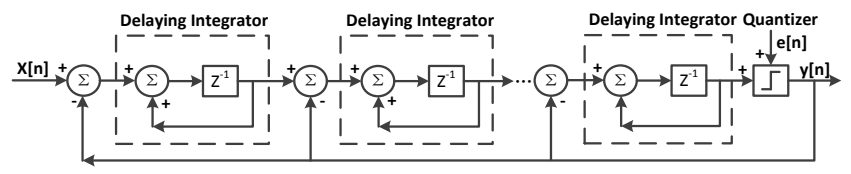

Figure 1. L-order Sigma-Delta modulator linear model

The transfer function Y, the noise transfer function NTF and the signal transfer function STF as follows

$$
\begin{gathered}
Y(z)=z^{-L} X(z)+\left(1-z^{-1}\right)^{L} E(z) \\
N T F(z)=\left(1-z^{-1}\right)^{L} \\
\operatorname{STF}(z)=z^{-L}
\end{gathered}
$$

According to the theory of signals and systems, signal and noise amplitude frequency response is given by

$$
\begin{gathered}
|\operatorname{STF}(f)|=1 \\
|\operatorname{NTF}(f)|=\left[2 \sin \left(\frac{\pi f}{f_{s}}\right)\right]^{L}
\end{gathered}
$$

So, L-order Sigma-Delta modulator for signal is an all pass filter, and for the quantization noise is a high pass filter.

The NTF and STF curves of L-order sigma-delta modulator are shown in Fig.3. Therefore, the Sigma-Delta modulator has the obvious characteristics of the noise shaping. With the L increased, the noise amplitude at low frequencies is lower, the signal-to-noise ratio of the modulator is higher, however, the amplitude of noise at high frequency is higher [5] [6] [7].

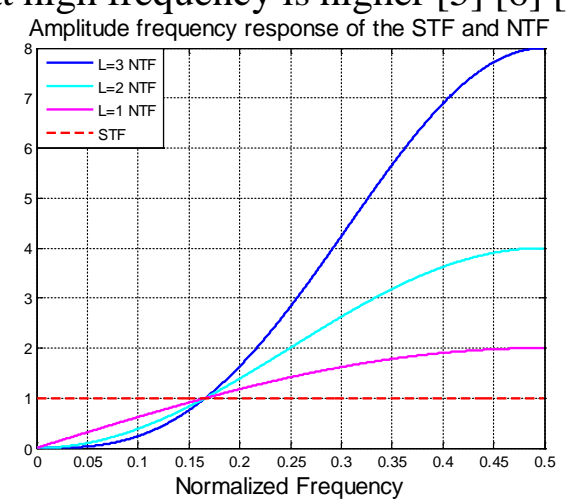

Figure 2. The NTF and STF curves of L-order sigma-delta modulator

The power spectral density of quantization noise as follows.

$$
\begin{aligned}
N_{y}(f) & =|\operatorname{NTF}(f)|^{2} N_{e}(f) \\
& =\left[2 \sin \left(\frac{\pi f}{f_{s}}\right)\right]^{2 L}\left(\frac{\Delta^{2}}{12}\right) \frac{2}{f_{s}} \\
& =\left[2 \sin \left(\frac{\pi f}{f_{s}}\right)\right]^{2 L} S_{B 0} \frac{2}{f_{S}}
\end{aligned}
$$


Where $N_{y}(f)$ is power spectral density, $\Delta$ is the quantization unit of ADC. $S_{B 0}$ is The total power of quantization noise and is a constant.

Assuming that the signal bandwidth is $f_{B}$ and $f_{B} \square f_{S}$, so the output quantization noise of the modulator in the band of energy and the output SNR are known.

$$
\begin{aligned}
& S_{B y}=\int_{0}^{f_{B}} N_{y}(f) d f \\
& =\int_{0}^{f_{B}}|N T F(f)|^{2} N_{e}(f) d f \\
& =\frac{2 S_{B 0}}{f_{S}} \int_{0}^{f_{B}}\left[2 \sin \left(\frac{\pi f}{f_{S}}\right)\right]^{2 L} d f \\
& \underline{\underline{f \square f_{s}}} \frac{2 S_{B 0}}{f_{S}} \int_{0}^{f_{B}}\left(\frac{2 \pi f}{f_{S}}\right)^{2 L} d f \\
& =\frac{\pi^{2 L}}{2 L+1}\left(\frac{2 f_{B}}{f_{S}}\right)^{2 L+1} S_{B 0} \\
& =\frac{\pi^{2 L} S_{B 0}}{(2 L+1)(O S R)^{2 L+1}} \\
& S N R=10 \log \left(\frac{P_{i}}{S_{B y}}\right) \\
& =10 \log \left[\frac{(2 L+1) \times P_{i} \times O S R^{2 L+1}}{\pi^{2 L} \times S_{B 0}}\right] \\
& =10 \log \left(P_{i}\right)-10 \log \left(S_{\text {B0 }}\right)+ \\
& 10(2 L+1) \log (\text { OSR })+10 \log \left(\frac{2 L+1}{\pi^{2 L}}\right)
\end{aligned}
$$

Where $\operatorname{OSR}=f_{\mathrm{s}} / 2 f_{\mathrm{B}}$ is over sampling.

From the above two equation, $S_{B y}$ is inversely proportional to the sampling rate of $\mathrm{OSR}^{\wedge}(2 \mathrm{~L}+1)$. The SNR in the signal band increased with the sampling rate OSR and the order $\mathrm{L}$ increasing. However, for the higher order $(\mathrm{L}>2)$ Sigma-Delta modulator, the order will make the modulator unstable. For the implementation of the actual circuit, the non-ideal factors will affect the stability further [5]. So in actual design, the input amplitude of each integrator needs strict control, and the transfer function zeros and poles of NTF and STF need to consider carefully.

B. Simulation for The $2^{\text {nd }}$ order Sigma-Delta Modulator

In order to analyze the Sigma-Delta modulator further, using the Simulink toolbox to simulate the ideal model of the $2^{\text {nd }}$ order Sigma-Delta modulator [8]. The simulation model is shown in Fig. 3.

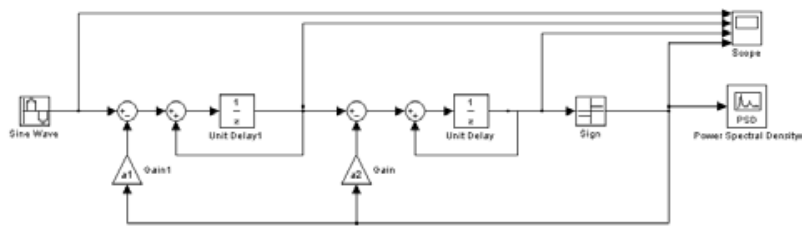

Figure 3. The simulation model of $2^{\text {nd }}$ order sigma-delta modulator

From the Fig. 3, the model consists of two basic delay units and a comparator sign. Where sine wave is a $1 \mathrm{kHz}$ sinusoidal signal, the normalized amplitude is 0.9 and the sampling rate is $2 \mathrm{MHz}$.

The power spectrum analysis of the output data flow signal is shown in Fig. 4, where the FFT point is $2^{\wedge} 19$, a fast Fourier transformation is applied. The figure results indicates that the $2^{\text {nd }}$ order Sigma-Delta modulator has obviously noise shaping effect, the quantization noise is removed to high frequencies band, and we can use a simple low-pass filter to get a high output SNR. 


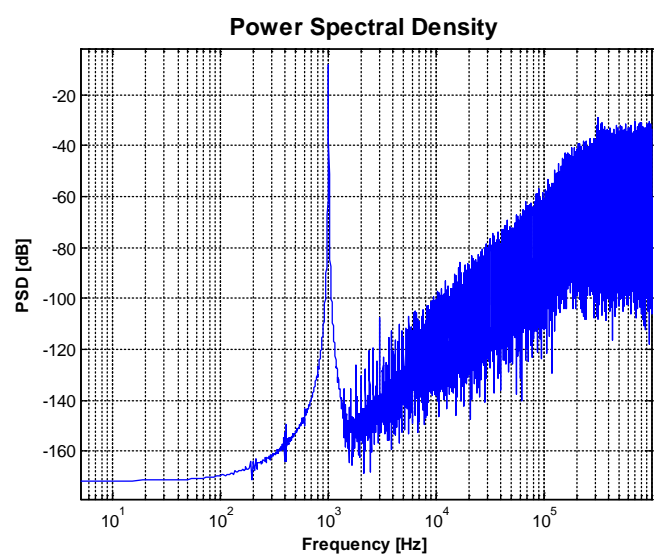

Figure 4. The output spectrum of the $2^{\text {nd }}$ order sigma-delta modulator

\section{The class $\mathbf{D}$ acoustic amplifier}

Usually a typical class D acoustic power amplifier is shown in Fig. 5. The first stage is the modulation part, which convert the input acoustic signal to a 1bit data stream. At present, the modulation method is commonly adopted PWM modulation. The second stage is power output part. the half-bridge and full-bridge are commonly used for the power output stage topology. The third stage is the matched filter stage, because of the transducer characteristics, a match filter network needs to use between the power amplifier and transducer for impedance matching.

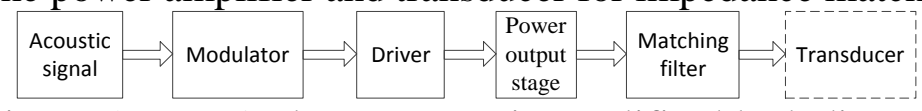

Figure 5. $\quad$ A class D acoustic amplifier block diagram

In order to implement the distance requirements of the sonar system, the power amplifier should provide several hundred volts to several thousand volts to the transducer, Therefor a transformer is always used in the acoustic amplifier.

In the transformer design, an adequate power capacity and frequency respond is needed. When transmit the modulated signal (Sigma-Delta signal or PWM signal), because of the abundant frequency components, the transformer needs a wide bandwidth. When the transformer working at high frequency, it should pay attention to set the skin effect which reduce the system efficiency.

Therefore the transformer need to carefully considered, in order to fit the power, efficiency, frequency response of the sonar system.

A. Matching Circuit for Transducer

Usually the transducer made by piezoelectric ceramics. The acoustic transducer is capacitive, its impedance varies with frequency. If the power amplifier drives the transducer directly, the amplifier will cause energy loss. The impedance matching is intended to make the transducer become a pure resistance device [9].

Usually, the piezoelectric transducer can be regarded as a lumped parameter system near the resonance frequency. the equivalent circuit is shown in Fig. 6(a). Where $C_{0}$ is the static capacitance, $R_{f}$ is mechanical resistance, $L_{f}$ is dynamic inductance, $C_{f}$ is dynamic capacitance.

When the transducer work in the resonant frequency, $L_{f}$ and $C_{f}$ series resonance, so the equivalent circuit can be simplified.it is shown in Fig. 6(b) diagram.

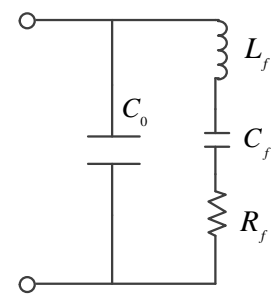

(a)

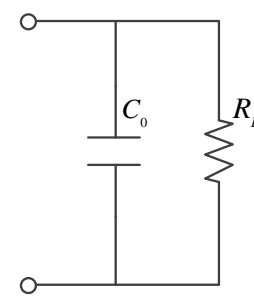

(b)

Figure 6. The equivalent circuit of transducer:(a) The dynamic equivalent circuit (b) The parallel equivalent circuit 
According to the equivalent circuit of transducer, the impedance analyzer could get the impedance data for a transducer. In engineering, series matching and parallel matching are often adopted to complete the matching circuit design. Fig. 7 gives the series matching circuit.

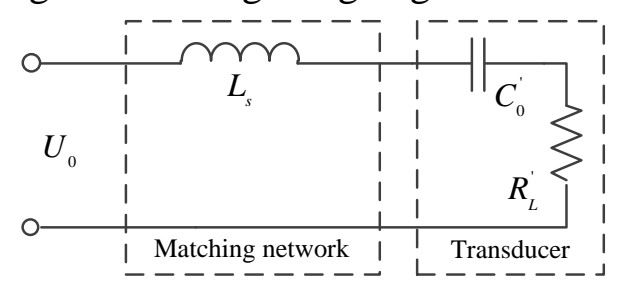

Figure 7. The series matching circuit of transducer

First, convert the transducer from parallel circuit to a series circuit. The relationship between the element values is given by

$$
\begin{aligned}
R_{L}^{\prime} & =\frac{R_{L}}{1+\left(\omega_{s} C_{O} R_{L}\right)^{2}} \\
C_{O}^{\prime} & =\frac{1+\left(\omega_{s} C_{O} R_{L}\right)^{2}}{\omega_{S}^{2} C_{O} R_{L}^{2}}
\end{aligned}
$$

Where $\omega_{s}$ is the angular frequency at the series resonance frequency.

The input impedance of equivalent circuit is given by

$$
Z=R_{L}^{\prime}+j\left(\omega_{S} L_{S}-\frac{1}{\omega_{S} C_{o}^{\prime}}\right)
$$

Make the imaginary part of impedance is zero, the value of matching inductance and equivalent resistance can be calculated.

$$
\begin{gathered}
L_{S}=\frac{1}{\omega_{S}^{2} C_{O}^{\prime}}=\frac{C_{o} R_{L}^{2}}{1+\left(\omega_{s} C_{o} R_{L}\right)^{2}} \\
R_{S}=R_{L}^{\prime}
\end{gathered}
$$

\section{The test results of the Sigma-Delta modulation amplifier}

In order to complete the application test for Sigma-Delta modulation amplifier and complete a comparative research with PWM modulation amplifier, a general acoustic amplifier circuit is designed which combined with the matching theory. Fig.8 shows the circuit schematic diagram.

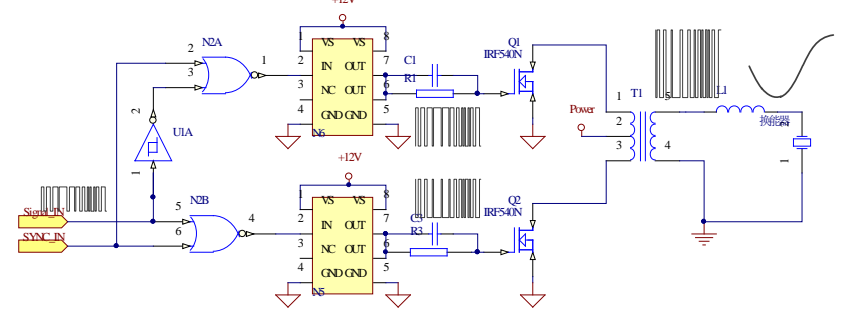

Figure 8. The circuit schematic diagram of the acoustic amplifier

The main circuit is a push-pull power amplifier with transformer coupled, using a series inductance to match the transducer. At the same time, high speed gate circuit to shape and protect the modulation signal, drive circuit is a special chip to drive the MOSFETs work efficiently, reduce the switching losses of the MOSFETs.

The Sigma-Delta modulation signal and PWM modulation signal are produced by MATLAB, the detail simulation parameters of two modulation methods is shown in table I. When the class D power amplifier sends the modulation signals, the maximum switching frequency is limited by the junction capacitance of the MOSFETs, and it cannot be too high when using. Therefore, the sampling frequency of the modulation signal cannot too large, avoid increase the switching losses, reduce the system efficiency. Considering the stability of the high order sigma-delta modulator in the actual constructed, using the 2nd order Sigma-Delta modulator which simulated in Part2 to create the Sigma-Delta modulation signal. 
TABLE I. THE PARAMETERS OF THE TWO MODULATIONS

\begin{tabular}{|c|c|c|}
\hline Modulation & The input signal frequency (kHz) & Normalized amplitude \\
\hline PWM. & $8-16$ & 0.8 \\
\hline Sigma-delta. & $8-16$ & 0.8 \\
\hline Modulation & Triangular wave frequency(kHz) & Sampling frequency(MHz) \\
\hline PWM. & 100 & 2 \\
\hline Sigma-delta. & -- & 2 \\
\hline
\end{tabular}

The modulated signal is written to the Angilent33522 function generator, the amplifier transmit the signal. Use AngilentDSO7034B to storage the measurement waveform. The driving signal is 10 $\mathrm{kHz}$ sine signals. Fig.9 is the driving signal spectrum with sigma-delta modulation. Fig.10 is the driving signal spectrum with PWM modulation

The Sigma-Delta modulation signal have an obviously noise shaping effects, the quantization noise in base band shifted to the high frequency.

Compared with the two modulation signal, The output SNR of the two modulation is basically the same. In the base band about $0 \mathrm{~Hz}$ to $50 \mathrm{kHz}$, the Sigma-Delta modulation signal effectively restrains the harmonic components of the modulating signal, so the output signal distortion can be improved.

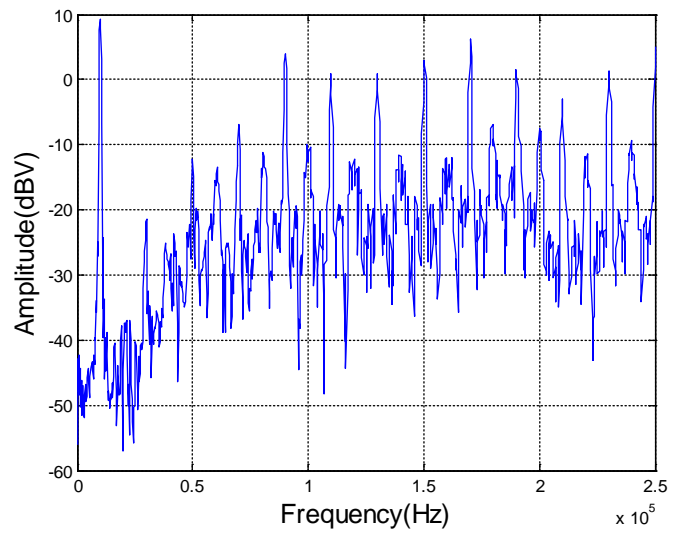

Figure 9. The output spectrum of sigma-delta drive signal

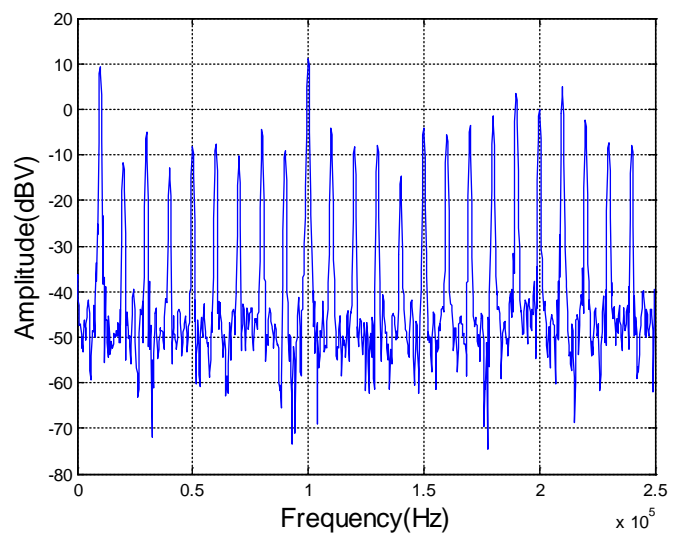

Figure 10. The output Spectrum of PWM drive signal

The final output signal spectrum after match filtering is shown in Fig. 11 and Fig. 12. For the same matching filter conditions, both of the two modulation signal have the same output SNR about $60 \mathrm{~dB}$. However, compared to the PWM modulation, the Sigma-Delta modulation can reduce the $2^{\text {nd }}$ harmonic about $3.5 \mathrm{~dB}$, and reduce the $3^{\text {rd }}$ harmonic about $20 \mathrm{~dB}$. 


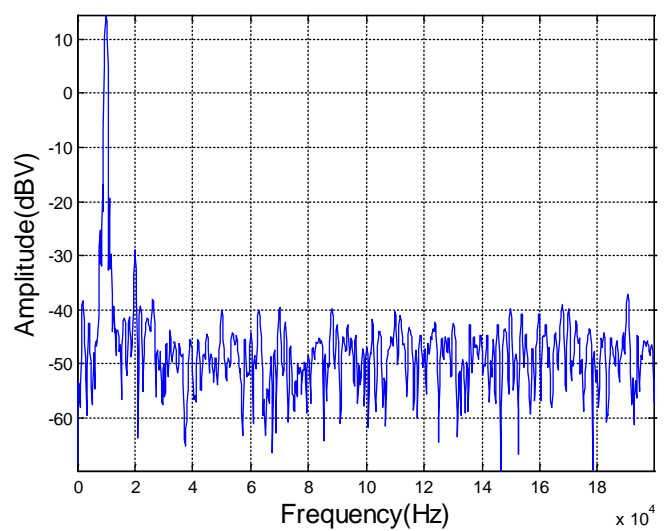

Figure 11. The output Spectrum of the filtered signal with sigma-delta modulator

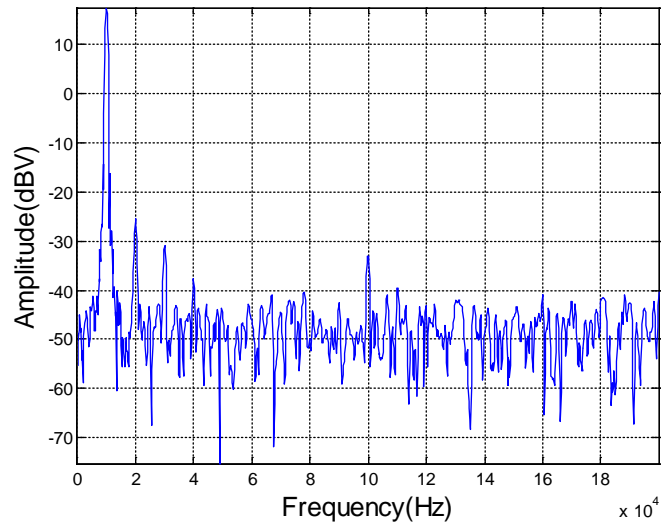

Figure 12. The output Spectrum of the filter signal with PWM modulator

Fig.13 shows the THD cure of the two modulation signal with different frequencies. The sigmadelta modulator has a good performance than the PWM modulator.

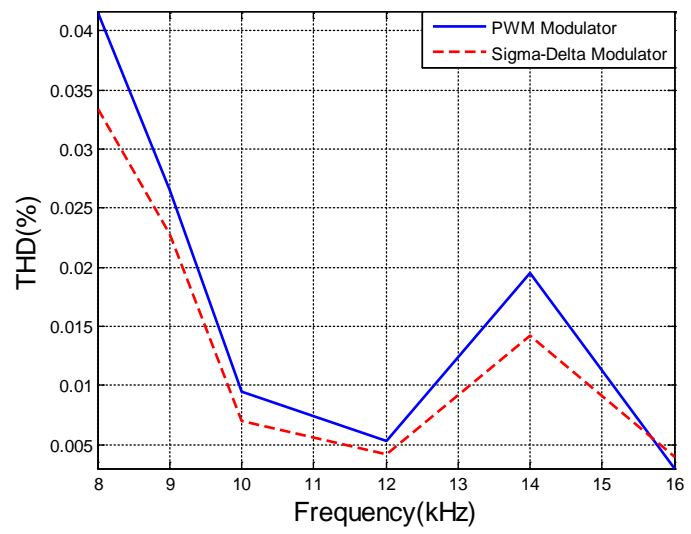

Figure 13. The THD curve of the two modulator

With the input signal is $10 \mathrm{kHz}$, the output power is $20 \mathrm{~W}$, the efficiency of the Sigma-Delta modulation amplifier is $81.6 \%$, and the PWM modulation amplifier is $83.2 \%$, Two kinds of modulation have high working efficiency.

In the actual test, the work efficiency and harmonic distortion of the Sigma-Delta modulation power amplifier is affected by system parameters seriously, especially the parameters of the matching filter circuits and transformer. In actual design for underwater acoustic power amplifier, the selection of modulation mode should be considered between the performance of the system and the difficulty of implementation.

\section{Conclusion}

This paper is briefly discussed the theory of Sigma-Delta modulator, analyzed the performance of L-order single bit Sigma-Delta modulator. A system modeling for $2^{\text {nd }}$ order Sigma-Delta modulator 
was proposed by Simulink. Under laboratory conditions, the acoustic amplifier with Sigma-Delta modulation and PWM modulation was constructed, combined the matching theory, the impedance matching with acoustic transducer is implemented. The test results show that: the Sigma-Delta modulation technique can be used for acoustic amplifier, Compared with PWM modulator, SigmaDelta modulator has the features of high output SNR, high work efficiency and low THD.

\section{References}

[1] Zhi Shaolong, Yuan Zhaokai, Li Yu, Yin Li, Huang Haining, "Miniaturized underwater acoustic signal modulation and transmission system,” Chinese Journal of Scientific Instrument, vol. 33, no. 7, 2012, pp. 1668-1675.

[2] A. Sanchez, S. Blanc, P. Yuste, J.J. Serrano, "A low cost and high efficient acoustic modem for underwater sensor networks,” OCEANS, 2011 IEEE, Spain, 6-9 June 2011, pp. 1-10.

[3] Xiaoka Xu, Shengli Zhou, "Study of Class-D Power Amplifiers for Underwater Acoustic OFDM Transmissions,” Oceans - San Diego, 2013, pp. 1-3

[4] César Pascual, Zukui Song, "High-Fidelity PWM Inverter for Digital Audio Amplification Spectral Analysis, Real-Time DSP Implementation and Results,” IEEE Transactions on power elctronics, vol.18, no1, January 2003

[5] R. Schreier, G.C.Temes, “Understanding Delta-Sigma Data Converters,” Piscataway, IEEE Press, 2005.

[6] Steven R. Norsworthy, Richard Schreier, Gabor C. Temes, Delta-sigma Data Converters: Theory, Design, and Simulation. New York: IEEE Press, 1996.

[7] Hein S, Zakhor A, “On the stability of sigma delta modulators,” IEEE Transactions on Signal Process, vol. 41, Issue. 7, 1993, pp. 2322-2348.

[8] SIMULINK and MATLAB User’s Guides, The MathWorks, Inc., 2002

[9] Yu Hongpei, Yuan Chongyao, "The Impedance Matching About the Transducer and transmitter,” Technical Acoustics, vol.10, no. 1, 1991, pp.22-28. 